\title{
Isolated Leptons at HERA
}

\author{
Gerhard Brandt ${ }^{1}$ \\ 1- Deutsches Elektronen-Synchrotron \\ Notkestr. 85, 22761 Hamburg - Germany
}

\begin{abstract}
Events with high energy isolated leptons (electrons and muons) and missing transverse momentum are studied using the full $e^{ \pm} p$ data samples collected by the H1 and ZEUS experiments at HERA, corresponding to a total integrated luminosity close to $1 \mathrm{fb}^{-1}$. Within the Standard Model, events with isolated leptons and missing transverse momentum mainly originate from the production of single $W$ bosons. The total single $W$ boson production cross section is measured. The analyses are completed by studies of the dynamics of single $W$ production and a search for isolated tau leptons.
\end{abstract}

\section{Introduction}

In the years 1994-2007 electrons ${ }^{\mathrm{a}}$ and protons collided at centre-of-mass energies of up to $319 \mathrm{GeV}$ at HERA. The final integrated luminosity of about $0.5 \mathrm{fb}^{-1}$ per experiment gives access to rare processes with cross sections of the order $1 \mathrm{pb}$. The production of single $W$ bosons is such a rare process in the Standard Model (SM). In case of subsequent leptonic decay of the $W$ boson this process gives rise to events containing high energy leptons and missing transverse momentum. This topology is also particularly interesting as it may be a signature of physics beyond the Standard Model (BSM). In this talk [1] the analysis of events with isolated leptons and missing transverse momentum by the H1 [2] and ZEUS [3] experiments at HERA using the full data set is presented.

\section{Event Selection}

In both analyses, leptons (electrons or muons) with $P_{T}^{\ell}>10 \mathrm{GeV}$ are selected. The $\mathrm{H} 1$ search covers the lepton polar angle range $5^{\circ}<\theta_{\ell}<140^{\circ}$ and the ZEUS search $15^{\circ}<\theta_{\ell}<120^{\circ}$. Missing transverse momentum $P_{T}^{\text {miss }}>12 \mathrm{GeV}$ is required. The leptons are required to be isolated against the hadronic final state by a distance $R>1$ in $(\eta, \phi)$ space, where $\eta$ is the pseudorapidity and $\phi$ is the azimuthal angle.

A total of 53 events are observed in the full $e^{ \pm} p$ data sample by H1, in good agreement with the SM prediction of $54.1 \pm 7.4$. ZEUS correspondingly observe 40 events for a SM prediction of $48.0 \pm 5.9$. Figure 1 shows the hadronic transverse momentum $P_{T}^{X}$ distributions of the selected events, compared to the SM prediction, for the combined electron and muon channels separately for the $e^{+} p$ and $e^{-} p$ data samples as measured by the $\mathrm{H} 1$ and ZEUS experiments. For both experiments, the SM prediction is dominated by single $W$ production. SM-like events are concentrated at low values of $P_{T}^{X}$, while events untypical for SM processes are found at high values. In this region, in particular in the $e^{+} p$ data, $\mathrm{H} 1$ sees a slight excess of events corresponding to a significance of $2.4 \sigma$. This excess has been previously reported using $118 \mathrm{pb}^{-1}$ of data [4] and has decreased in significance with the addition of more data. Combined with the $e^{-} p$ data and the ZEUS data, the significance is further reduced.

\footnotetext{
an the following the term "electron" is used generically to refer to both electrons and positrons, unless otherwise stated.
} 

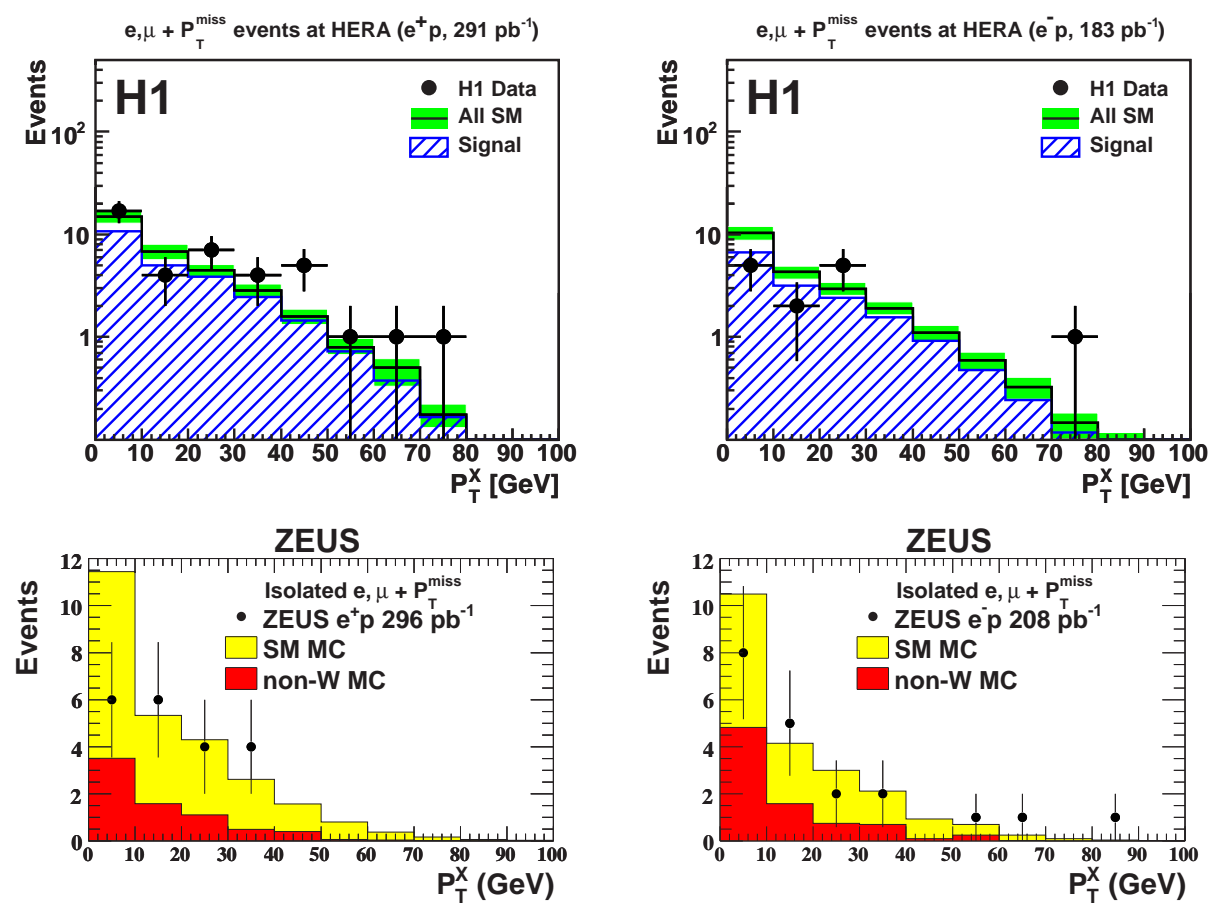

Figure 1: The hadronic transverse momentum $P_{T}^{X}$ distributions in the combined electron and muon channels for the $e^{+} p$ (left) and $e^{-} p$ (right) data samples measured by the H1 (top) and ZEUS (bottom) experiments. The data (points) are compared to the SM expectation. The signal component of the SM expectation, dominated by real $W$ production, is shown as the hatched (red) histogram.

Extensive studies to rule out an experimental origin of the excess due to not well understood backgrounds have been carried out. Backgrounds enter the final selection mainly due to misidentification of hadrons as leptons (in charged current events), or due to mismeasurement of missing energy (in neutral current events). These and all other background processes entering the final sample were studied by enriching contributions of the respective processes to the SM prediciton in dedicated control samples. The studies showed that all backgrounds contributing to the final sample are understood within the systematic and model uncertainties.

\section{Measurement of Single $W$ Production}

Based on this sample of isolated leptons, both $\mathrm{H} 1$ and ZEUS measure the single $W$ production cross section to $1.14 \pm 0.25$ (stat.) \pm 0.14 (sys.) pb, and $0.89_{-0.22}^{+0.25}$ (stat.) \pm 0.10 (syst.) pb, respectively. The measurements are in agreement with each other and with the SM expectation of $1.27 \pm 0.19 \mathrm{pb}$. The sample was also exploited for further studies of the dynamics of single $W$ production in ep collisions by H1. The sensitivity to triple gauge couplings at the $W W \gamma$ vertex was probed and range limits on the coupling parameters $\kappa$ 

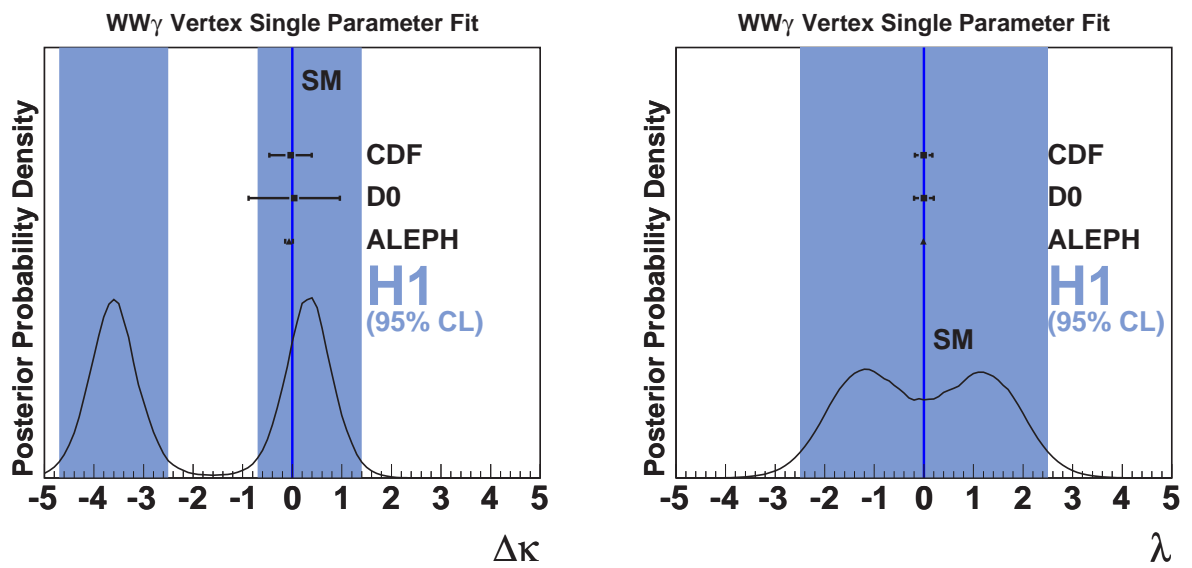

Figure 2: Probability distributions (in arbitrary units) of single parameter fits to (left) $\Delta \kappa$ and (right) $\lambda$. The obtained $95 \% \mathrm{CL}$ limits are shown (shaded areas) in comparison with those of D $\varnothing$ [6], CDF [7], and ALEPH [5]. The SM expectation values are also indicated.

and $\lambda$ derived. The resulting likelihood distribution is shown in figure 2. The constraints $-4.7<\Delta \kappa<-2.5$ or $-0.7<\Delta \kappa<1.4$, and $-2.5<\lambda<2.5$. were found. While not competitive to measurements at LEP [5], the constraints on $\Delta \kappa$ are comparable in sensitivity to measurements at the TeVatron $[6,7]$, and are complementary to both. The $W$ polarisation fractions, which are sensitive to the angular properties of the decay and may be different for SM and BSM contributions, are also probed. Figure 3 shows the results of a fit of the $W$ polarisation fractions. The sensitivity to differentiate $W$ production from SM and BSM processes is found to be at the 1-sigma level.

\section{Search for Isolated Tau Leptons}

A search for isolated tau-leptons complements the analysis of the electron and muon channels. In the SM, comparable results to the other channels are expected due to lepton universality, and in many BSM models, such as SUSY scenarios with a high $\tan \beta$, an abundance of tau leptons is expected in the final state. Hadronically decaying tau leptons are selected by looking for narrow jets with $P_{T}>7 \mathrm{GeV}$ in events with a missing transverse momentum of $P_{T}^{\text {miss }}>12 \mathrm{GeV}$. The tau candidates are required to be isolated against other objects by a distance of $R>1$. A track multiplitcity of one, corresponding to a 1-prong decay topology, is strictly enforced. Figure 4 shows the selected tau candidates as a function of the hadronic transverse momentum, not including the tau-jet candidate, $P_{T}^{X^{\prime}}$. The background situation is challenging, and the SM prediction for the final sample is dominated by charged current events. At high $P_{T}^{X^{\prime}}>25 \mathrm{GeV}$ one event is observed, for a prediction of $1.5 \pm 0.21$ with a signal contribution of $0.59 \pm 0.09$. In this analysis no signal for tau lepton production is observed at HERA. 


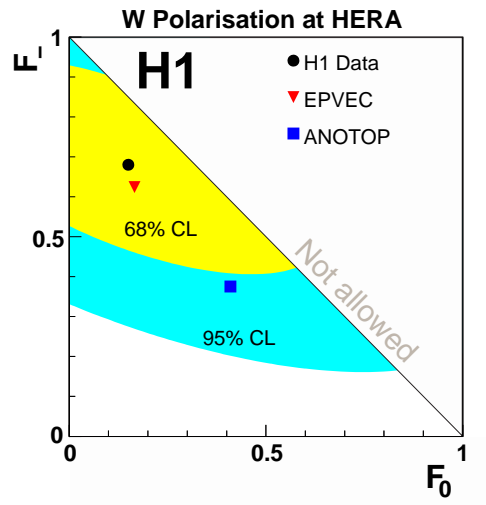

Figure 3: The plane showing the fit result for the simultaneously extracted left handed $\left(F_{-}\right)$and longitudinal $\left(F_{0}\right) W$ boson polarisation fractions (point) with the corresponding $68 \%$ and $95 \%$ CL contours. Also shown are the values of predictions from single $W$ production in the SM (EPVEC, triangle) and anomalous single top production (ANOTOP, square).

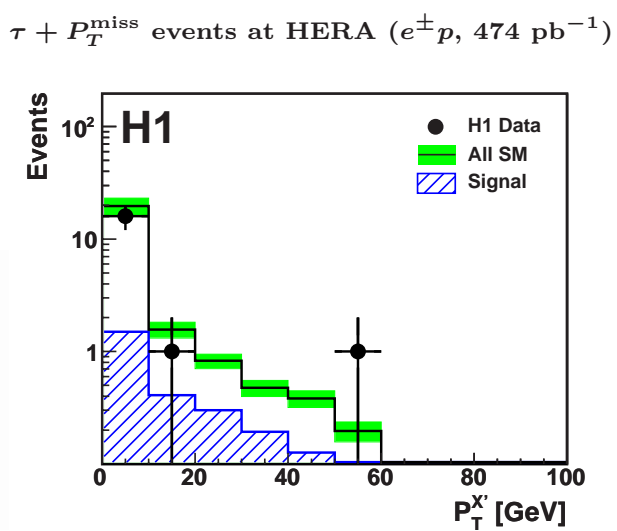

Figure 4: Distribution of the hadronic transverse momentum not including the tau-jet candidate $P_{T}^{X^{\prime}}$ in the tau channel for the $e^{ \pm} p$ data sample. The data (points) are compared to the SM expectation (open histogram). The signal component of the SM expectation is shown as the hatched histogram.

\section{Summary}

The analysis of events with isolated leptons and missing transverse momentum at HERA has been carried out by the $\mathrm{H} 1$ and ZEUS experiments on the full HERA data. Overall good agreement of the data with the SM prediction was found. With the addition of the measurement of the single $W$ production cross section and dynamics, and the search for isolated tau leptons a large part of the isolated leptons programme is complete.

\section{References}

[1] Slides:

http: //indico. cern. ch/contributionDisplay . py? contribId=33\&sessionId=2\&conf Id=53294

[2] F. D. Aaron et al. [H1 Collaboration], "Events with Isolated Leptons and Missing Transverse Momentum and Measurement of $W$ Production at HERA", arXiv:0901.0488 [hep-ex].

[3] S. Chekanov et al. [ZEUS Collaboration], "Search for events with an isolated lepton and missing transverse momentum and a measurement of W production at HERA", Phys. Lett. B 672 (2009) 106 [arXiv:0807.0589 [hep-ex]].

[4] V. Andreev et al. [H1 Collaboration], Phys. Lett. B 561 (2003) 241 [hep-ex/0301030].

[5] S. Schael et al. [ALEPH Collaboration], Phys. Lett. B 614 (2005) 7.

[6] V. M. Abazov et al. [DØ Collaboration], Phys. Rev. D 71 (2005) 091108 [hep-ex/0503048].

[7] T. Aaltonen et al. [CDF Collaboration], Phys. Rev. D 76 (2007) 111103 [hep-ex/0705.2247]. 